\title{
ESTRATEGIAS DE AFRONTAMIENTO (COPING) Y SU RELACIÓN CON EL LOGRO ACADÉMICO EN MATEMÁTICA Y LENGUA EN ADOLESCENTES DE NOVENO AÑO DE EDUCACIÓN GENERAL BÁSICA
}

\author{
Alicia Massone y Gloria González \\ Universidad Nacional de Mar del Plata, Argentina
}

\section{INTRODUCCIÓN}

Actualmente, en el campo educacional se le presta cada vez mayor atención a la evaluación de las estrategias de afrontamiento, debido al reconocimiento de la importancia que adquiere el fomento de éstas en los jóvenes, máxime si se contemplan las presiones y el estrés creciente al que están sometidos en ambientes de estudio y trabajo cada vez más competitivos. En este marco, intentando superar la visión de los adultos acerca de cómo enfrentan el estrés los adolescentes, se han hecho en los últimos años estudios que intentan dar cuenta de la conducta de afrontamiento de los adolescentes (Dise-Lewis, 1988; Compas, Malcarne y Fondacaro, 1988; Patterson y Mc. Cubbin, 1987; Stark y Williams, 1989) asumiendo que la cuestión que nos ocupa tiene importancia para toda la sociedad.

Asimismo, se viene reconociendo que la utilización de estas estrategias no es ajena a lo que acontece en el ámbito escolar ya que se relaciona con cuestiones tales como la obtención, el análisis y la organización de la información; la comunicación de ideas e informaciones; la planificación, el trabajo en equipo y la resolución de problemas o cómo buscar el consejo de los demás o hablar de los propios problemas con otros.

En este sentido, es dable esperar que se haga consciente en los educadores la importancia de su mediación para el desarrollo de la capacidad para el afrontamiento, entendida como estrategia cognitivoconductual, con miras al logro de una adaptación y transición efectiva hacia la adultez. Los educadores pueden ayudar a mejorar la capacidad de afrontamiento de los estudiantes tanto comprendiendo y dando respuesta a sus necesidades en el ámbito de la educación curricular como mediante programas educacionales especiales.

En el caso del estudio que nos ocupa se ha utilizado como instrumento de evaluación del afrontamiento en adolescentes la versión española del ACS, Escalas de Afrontamiento en Adolescentes, de Frydenberg y Lewis (1995), dado que es innovador desde la perspectiva de que no se limita a la observación externa sino que promueve la autorreflexión y que se le considera uno de los más completos para elucidar esta capacidad. Además, permite establecer una distinción entre afrontamiento general y específico, y resulta adecuado para promover el cambio conductual autodirigido y para favorecer las decisiones del grupo de estudiantes cuando toman conocimiento de los resultados de la evaluación. También, y dado que se ha reconocido su validez en el ámbito educativo como instrumento de discriminación entre estrategias de afrontamiento de los individuos o 
grupos que actúan en diferentes contextos, ha facilitado la comparación de los estudiantes que nos ocupan, los cuales se desarrollan en contextos altamente diferenciados donde se encara con diferentes grados de sistematización el trabajo sobre las estrategias de afrontamiento de los alumnos. En términos generales, parece no haber dudas acerca de que el reconocimiento de las estrategias que ponen en juego los adolescentes «echa luz» y aumenta la comprensión del maestro sobre la conducta de sus alumnos. En particular, se recomienda su utilización cuando existe estrés en el ámbito escolar o en la familia, en los últimos cursos de la escuela, para estudiar el clima social de la clase, para afianzar el conocimiento de la identidad de los jóvenes y en relación a otras variables como sexo, nivel socioeconómico, edad y origen étnico.

\section{METODOLOGÍA Y DESCRIPCIÓN DE INSTRUMENTOS}

Se trabajó con una muestra accidental de 447 adolescentes que asisten a noveno año de la educación general básica en seis escuelas publicas y dos privadas de la ciudad de Mar del Plata, de los cuales un 50,3\% son mujeres y un $49,7 \%$ varones y sus edades oscilaron de 15 a 17 años.

Se administraron las Escalas de Afrontamiento para Adolescentes (ACS, Frydenberg, E. y Lewis, R. 1996) y las pruebas de logro diseñadas por la Dirección General de Escuelas de la Pcia. de Buenos Aires.

El ACS es un inventario de autoinforme compuesto de 80 elementos, 79 de tipo cerrado y 1 de final abierto, que permiten evaluar con fiabilidad 18 estrategias de afrontamiento diferentes que se han identificado conceptual y empíricamente. Estas fueron elaboradas a partir del supuesto de que en la capacidad de afrontamiento pueden reconocerse estrategias diferentes que los sujetos utilizan con diferente fuerza. Los 18 factores que se reflejan en las estrategias de afrontamiento de los adolescentes son: buscar apoyo social, concentrarse en resolver problemas, esforzarse y tener éxito, preocuparse, invertir en amigos íntimos, buscar pertenencia, hacerse ilusiones, no afrontar, reducir la tensión, llevar a cabo acciones sociales, ignorar el problema, autoinculparse, reservar el problema para si, buscar apoyo espiritual, fijarse en lo positivo, buscar apoyo profesional, buscar diversiones relajantes y/o distraerse físicamente. Las anteriores dan lugar a tres estilos básicos de afrontamiento: dirigido a la resolución del problema, afrontamiento en relación con los demás y afrontamiento improductivo.

Para la evaluación de logro en Matemática y Lengua se aplicaron las Pruebas diseñadas por el Sistema de Medición y Evaluación de la Calidad Educativa de la Policía de Buenos Aires en el marco del Proyecto de diseño e implementación de un sistema provincial de medición y evaluación de la calidad educativa (1999). La característica esencial de los instrumentos es la de evaluar aprendizajes cognitivos de nivel superior, es decir, habilidades complejas orientadas a una meta. El puntaje máximo en Matemática es de 30 puntos y en Lengua es de 32 puntos. 
Las pruebas aplicadas evalúan contenidos específicos y determinadas competencias mediante una serie de ítems de respuesta cerrada y selección múltiple, compuestos por un enunciado y cuatro opciones o alternativas de las cuales solo una es correcta y las demás funcionarían como distractores orientados a relevar errores comunes en los que el alumno incurre con frecuencia.

La estructura de las pruebas de Matemática, considera cuatro dimensiones. Estas se han definido como:

1. Estructuras conceptuales

2. Procesos cognitivos

3. Procedimientos de trabajo para la resolución de problemas.

4. Componentes actitudinales.

En las pruebas para la evaluación del área Lengua, las dimensiones a evaluar son:

- El enfoque comunicativo de la enseñanza de la disciplina.

- El enfoque cognitivo de los procesos de aprendizaje.

- El enfoque de evaluación.

El instrumento se compone de un ítem de respuesta cerrada y de un ítem de producción escrita de tendencia abierta. Considera tres competencias comunicativas básicas: las que siguen los lineamientos curriculares de Provincia, a saber, escuchar, leer y escribir; las relacionadas con la dimensión del conocimiento de los textos funcionales, los literarios, y las producidos por los medios masivos de comunicación. A su vez, el conocimiento de los textos supone competencias particularizadas en función de la especificidad del tipo de textual (descripción, narración, argumentación) y la variedad genérica (crónica, publicidad, cuento, explicación de divulgación) de los que se trate.

\section{RESULTADOS}

Según los lineamientos generales de los datos se registraron diferencias muy significativas $(p<, 01)$ tanto en Matemática como en Lengua entre las escuelas públicas y privadas. En este sentido, se ha observado que la media de las escuelas públicas en Matemática es de 9,72/30 mientras que en las privadas es de 18,40/30. En el área de Lengua los puntajes obtenidos también demostraron diferencias significativas $(p<, 01)$; las escuelas públicas alcanzaron una media de 16,31/32 y las privadas de 24,58/32 respectivamente. En este sentido, y dadas las características del instrumento de evaluación, podríamos decir que los alumnos de escuelas públicas en el caso particular del área de Lengua poseen las competencias comunicativas básicas (leer-escucharescribir) en un nivel inferior de desarrollo en relación con los alumnos de las escuelas de gestión privada. Las 
diferencias significativas en el rendimiento en las áreas evaluadas podría deberse a que estos estudiantes están expuestos en gran medida a estímulos altamente ansiógenos y estresantes como la necesidad de trabajar, falta de contención familiar, elevada exposición a medios masivos de comunicación, que se traducen en escasa motivación para la tarea y en severas dificultades de comprensión y compromiso con la prueba.

Si entendemos que el puntaje máximo previsto por las pruebas se corresponde con la apropiación por parte de los alumnos de los contenidos conceptuales, procedimentales y cognitivos previstos en el currículo para noveno año de EGB 3, son preocupantes, en general, los resultados obtenidos. Sin embargo, debería prestarse especial atención a lo que ha ocurrido en las escuelas públicas donde los resultados en el área de Matemática se ubican alrededor del tercio de lo que los alumnos, al menos desde el supuesto a nivel curricular, deberían saber. La afirmación antedicha se ampararía en que para estos adolescentes, pertenecientes a sectores urbano-marginales, la escuela pública es tal vez el único enclave en el que podrían dotarse de las competencias básicas para un genuino ejercicio de la ciudadanía.

Si se analiza el nivel de logro según la variable tipo de gestión es dable sostener que las escuelas de gestión privada obtuvieron resultados que se hallan por encima del $50 \%$ de los conocimientos escolares que, se supone, deben haber adquirido los alumnos de noveno año de E.G.B. 3. En lo referente a las escuelas de gestión pública, la situación de mayor déficit pareciera concentrarse en el área Matemática, ya que los resultados rondan el $30 \%$ de las competencias educativas previstas para estos alumnos. Si destacamos que las pruebas en cuestión evalúan contenidos mínimos los resultados observados en la tabla 1 darían cuenta de la gravedad de los déficits existentes.

De los resultados obtenidos en la escala ACS. se deduce que la forma preferida de afrontar los problemas cotidianos y escolares de nuestros adolescentes son: Buscar diversiones relajantes (Dr), en la que alcanzaron la media más alta $(74,5208)$, se caracteriza por elementos que describen actividades de ocio como leer, o pintar. Le siguen Distracción física (Fi), con 71,4194, trata de elementos que se refieren a hacer un deporte o actividad; Invertir en amigos íntimos (Ai) con 69,71 induce a la búsqueda de relaciones personales intimas y finalmente con una media similar que ronda en los 68,00 Preocuparse $(P \eta)$, Buscar pertenencia $(P e)$, y Fijarse en lo positivo (Po).

Las estrategias menos usadas por los adolescentes en estudio fueron, Reducción de tensión (Rt), que se caracteriza por elementos que reflejan un intento de sentirse mejor y relajar la tensión y Acción social (As) que se refiere al hecho de dejar que otros conozcan el problema y tratar de conseguir ayuda $u$ organizar actividades.

Las correlaciones obtenidas entre puntaje en Matemática y Lengua y estrategias de afrontamiento se presentan a continuación en la siguiente tabla: 


\begin{tabular}{|c|c|c|c|c|c|c|}
\hline ESTRATEGIAS & & $\begin{array}{l}\text { concentrarse } \\
\text { en resolver } \\
\text { el problema }\end{array}$ & $\begin{array}{c}\text { buscar } \\
\text { pertenencia }\end{array}$ & $\begin{array}{l}\text { Acción } \\
\text { social }\end{array}$ & $\begin{array}{c}\text { buscar } \\
\text { apoyo } \\
\text { espiritual }\end{array}$ & $\begin{array}{c}\text { buscar } \\
\text { apoyo } \\
\text { profesional }\end{array}$ \\
\hline MATEMÁTICA & $\begin{array}{l}\text { Pearson } \\
\text { Correlación }\end{array}$ &, $390^{\star *}$ &, $102^{*}$ & $-161^{* *}$ &,$- 161^{\star *}$ &,$- 126^{* *}$ \\
\hline LENGUA & $\begin{array}{l}\text { Pearson } \\
\text { Correlación }\end{array}$ &, $378^{\star \star}$ &, $134^{\star *}$ &,$- 205^{\star \star}$ &,$- 188^{* *}$ &,$- 129^{\star \star}$ \\
\hline
\end{tabular}

De las 18 estrategias que evalúa el instrumento sólo 5 correlacionan en forma significativa con los puntajes obtenidos por los adolescentes en Matemática y Lengua.

Pareciera que las habilidades de afrontamiento significativas a la hora de realizar actividades de Matemática y Lengua son, concentrarse en resolver el problema: estrategia dirigida a resolver el problema, que lo estudia sistemáticamente y analiza los diferentes puntos de vista. Buscar pertenencia: indica la preocupación del adolescente por sus relaciones con los demás y por lo que los otros piensan. En cuanto a las estrategias de acción social: se refiere al hecho de dejar que otros conozcan el problema y tratar de conseguir ayuda. buscar apoyo espiritual: empleo de la oración y creencias en la ayuda de un ser superior. Finalmente, buscar apoyo profesional: es la estrategia consistente en buscar apoyo de profesionales, maestros o consejeros, se obtuvieron correlaciones muy significativas de tipo negativo, que indicarían que aquellos adolescentes que tienen bajo puntajes en Matemática y Lengua, usan habitualmente estrategias de búsqueda de apoyo espiritual y profesional y de acción social a la hora de afrontar sus problemas.

Estas últimas correlacionan en sentido negativo, nos permitirían considerar que aquellos alumnos que buscan más apoyo social son los que estarían mas preocupados por su propia problemática y descuidan lo relativo a su desempeño en la escuela, obteniendo así bajo desempeño en las dos áreas. Notamos en este resultado la expresión de la personalidad adolescente que está centrado en su problemática y necesita apoyo para resolverla. Sobredimensiona su propia conflictiva y resta importancia a los aspectos académicoeducativos.

En este sentido, parecería que los adolescentes que más capacidad para concentrarse en resolver la tarea y que se inclinan por buscar grupos de pertenencia alcanzarían mejor desempeño (puntajes mas altos) en Matemática y Lengua. 


\section{CONCLUSIONES}

Los resultados obtenidos permiten establecer algunas interpretaciones referidas al modo en que pueden verse asociadas las estrategias de afrontamiento de los alumnos y su rendimiento académico en dos áreas curriculares axiales como son Lengua y Matemática.

Del análisis de los datos cabe concluir que hay ciertas estrategias que correlacionan positivamente con el nivel de logro sea cual sea la pertenencia a establecimiento educativo. Esto es, se hallaron correlaciones significativas entre nivel de logro y estrategias de afrontamiento que suponen apoyo social y afrontamiento en relación a los demás. Es decir, buscar grupos de pertenencia, revelar a los demás las preocupaciones y requerir apoyo espiritual y/o profesional.

Estos resultados podrían ser importantes para revitalizar la importancia del afrontamiento social de nuestros adolescentes y ser usados como insumos para algunos replanteos de la práctica áulica cotidiana, específicamente en lo referido a transmisión de contenidos en Matemática y Lengua.

Si encuadramos estos resultados en la consideración de que hace a la naturaleza del afrontamiento tender a la autorregulación y que el primer paso de ésta es la toma de conciencia, podría pensarse coincidiendo con Evans (1995) que los sujetos que han tenido mayores posibilidades de conocerse a sí mismos son los más favorecidos para trasladar este conocimiento al campo educativo, entre otros, facilitando que puedan conocer su conducta y adoptar aquellos cambios que resulten necesarios. En este estudio son precisamente los alumnos que han accedido a un espacio curricular dedicado al autoconocimiento aquellos en los que se aprecia la utilización de estrategias de afrontamiento más maduras y eficaces que las disponibles para el grupo de jóvenes que asisten a una escuela estatal que atiende a sectores sociales menos favorecidos. Es para es este último grupo de jóvenes especialmente para el que, entendemos, la escuela debería instituirse en un espacio real de adquisición y desarrollo de estrategias de afrontamiento eficaces y maduras. Hoy día se sabe que cuando los jóvenes son incapaces de afrontar sus problemas el impacto recae sobre la sociedad toda y que ayudar a los adolescentes a desarrollar estrategias sanas es estar trabajando a avor de un adulto que será capaz de afrontar adecuadamente sus problemas.

\section{REFERENCIAS BIBLIOGRÁFICAS}

COMPAS, B.; MALLAREN, V. L., y FONDACARO, K.M (1988): «Coping with stressful eventes in older childrens and young adolescents», en: Journal of Consulting and Clinical Psychology, vol. 6, pp. 405-411.

DISELEWIS, J.E. (1988): «The Life Events and Coping Inventory: an assessment of stress in children», en: Psychosomatic Medicine, vol. 50 (5), pp. 484-499 .

EVANS, G. (1995): Manual de Escalas de Afrontamiento para Adolescentes. Madrid, TEA. 
FRYDEMBERG, E. (1989): "Concerns of youth and how they cope: a study of Australian adolescents", unpublished doctoral dissertation. Melbourne, La Trobe University.

FRYDEMBERG, E., y LEWIS, R. (1990): «How adolescents cope with different concerns: The development of the Adolescent Coping Checklist (ACC)», en: Psychological Test Bulletin, vol.3 (2), pp. 63-73.

FRYDEMBERG, E., y LEWIS, R. (1991 a): «Adolescent coping. The different ways in which boys and girls cope», en: Journal of Adolescent, vol. 14, pp. 119-133.

FRYDEMBERG, E., y LEWIS, R (1991 b): «Australian adolescent coping styles», en: Australian Educational Researcher, vol. 18 (2), pp. 65-82.

MINISTERIO DE CULTURA Y EDUCACIÓN (2000): «Sistema Nacional de Evaluación de la Calidad Educativa», en: Manual de Evaluación área Matemática y Lengua. Buenos Aires.

PATTERSON, J. M., y MC. CUBBIN, H. I. (1987): «Adolescent coping style and behaviours: Conceptualisation and measurement», en: Journal of Adolescent, vol. 10, pp. 163-186

SPIRITO, A.; STARK, L. J., y WILLIAMS, C. A. (1989): «Development of a brief coping scales for use with Pediatric populations», en: Journal of Pediatric Psychology, vol. 13 (4), pp. 555-574. 


\title{
Contactar
}

Revista lberoamericana de Educación

\author{
Principal OEI
}

